\title{
Corrigendum
}

\section{Corrigendum to "Fatal Systemic Vasoconstriction in a Case of Metastatic Small-Intestinal NET"}

\author{
Jochen Stenzel, ${ }^{1}$ Sebastian Noe, ${ }^{2}$ Konstantin Holzapfel, ${ }^{3}$ \\ Franziska Erlmeier, ${ }^{4}$ and Florian Eyer ${ }^{1}$
}

${ }^{1}$ Department of Clinical Toxicology, Klinikum rechts der Isar, Technical University of Munich, Ismaningerstrasse 22, 81675 Munich, Germany

${ }^{2}$ Department of Internal Medicine II (Gastroenterology), Klinikum rechts der Isar, Technical University of Munich, Ismaningerstrasse 22, 81675 Munich, Germany

${ }^{3}$ Department of Diagnostic and Interventional Radiology, Klinikum rechts der Isar, Technical University of Munich, Ismaningerstrasse 22, 81675 Munich, Germany

${ }^{4}$ Institute of Pathology, Klinikum rechts der Isar, Technical University of Munich, Ismaningerstrasse 22, 81675 Munich, Germany

Correspondence should be addressed to Jochen Stenzel; j.stenzel@tum.de

Received 16 November 2017; Accepted 21 November 2017; Published 3 December 2017

Copyright (C) 2017 Jochen Stenzel et al. This is an open access article distributed under the Creative Commons Attribution License, which permits unrestricted use, distribution, and reproduction in any medium, provided the original work is properly cited.

In the article titled "Fatal Systemic Vasoconstriction in a Case of Metastatic Small-Intestinal NET" [1], the first and last names of all the authors were reversed. The revised authors' list is shown above and updated in place.

\section{References}

[1] J. Stenzel, S. Noe, K. Holzapfel, F. Erlmeier, and F. Eyer, "Fatal systemic vasoconstriction in a case of metastatic smallintestinal NET," Case Reports in Gastrointestinal Medicine, vol. 2017, Article ID 9810194, 6 pages, 2017. 


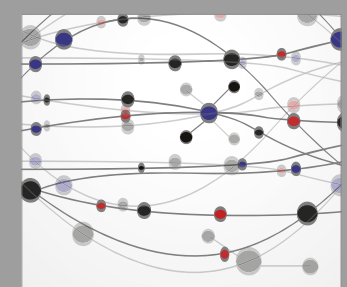

The Scientific World Journal
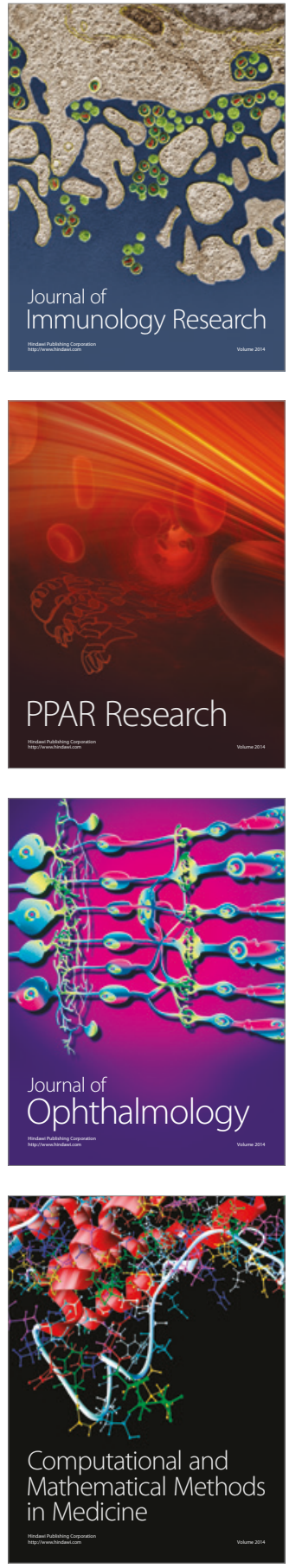

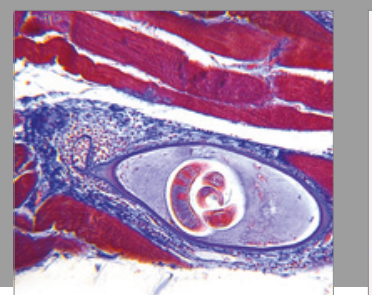

Gastroenterology Research and Practice
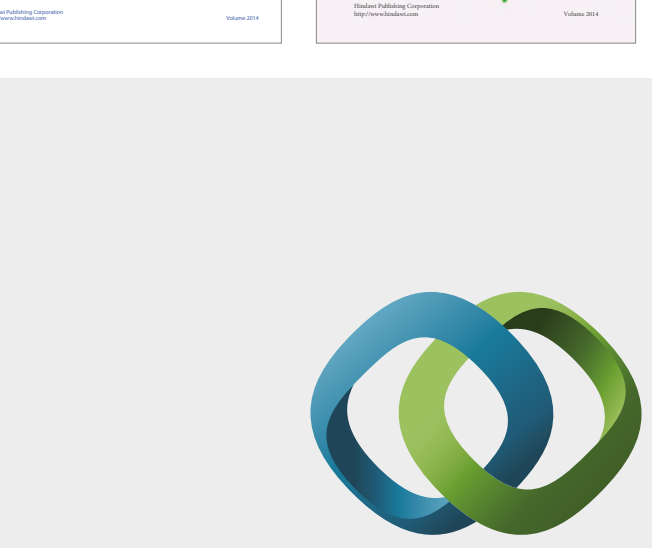

\section{Hindawi}

Submit your manuscripts at

https://www.hindawi.com
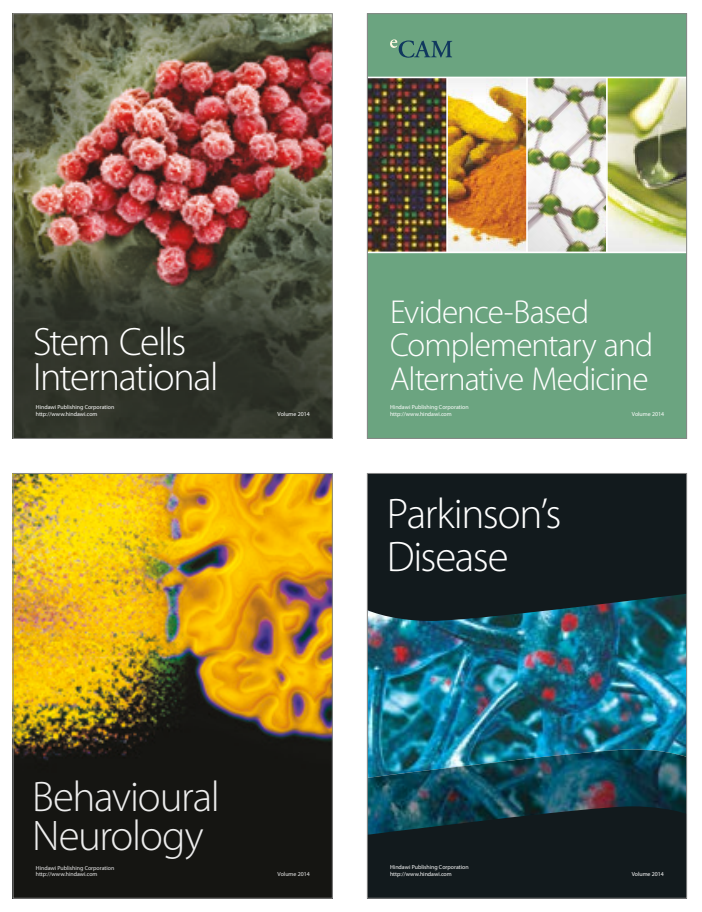
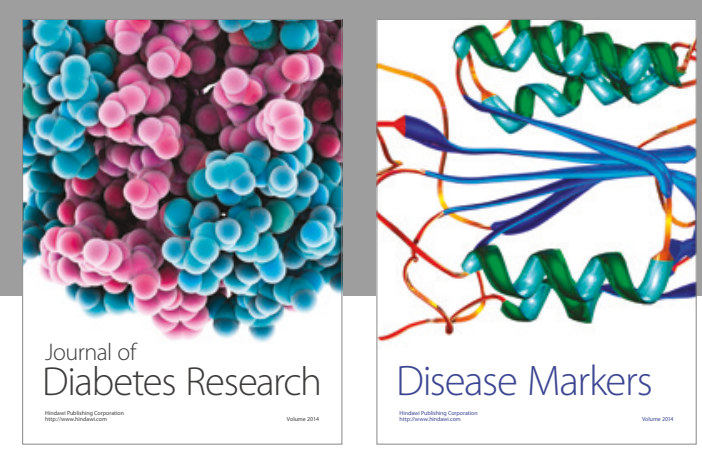

Disease Markers
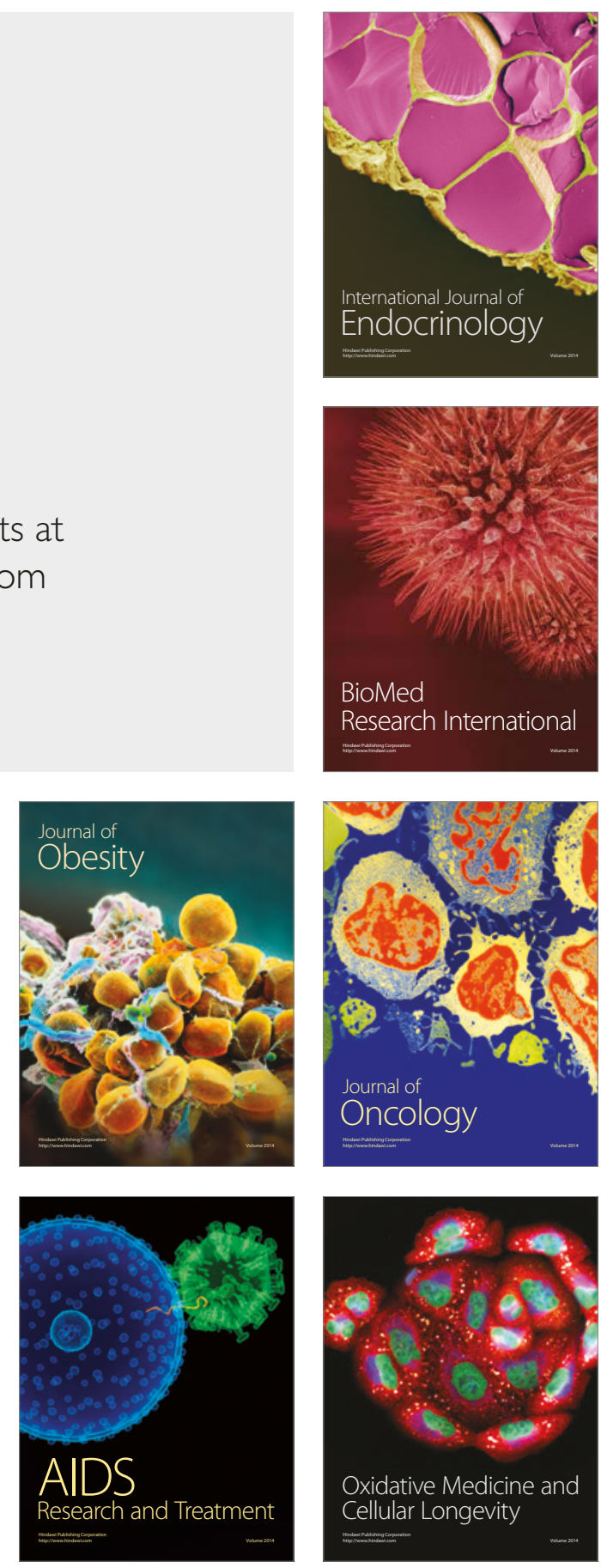